\section{ORIGINAL RESEARCH}

A. Mike

B.I. Glanz

P. Hildenbrand

D. Meier

K. Bolden

M. Liguori

E. Dell'Oglio

B.C. Healy

R. Bakshi

C.R.G. Guttmann

\title{
Identification and Clinical Impact of Multiple Sclerosis Cortical Lesions as Assessed by Routine 3T MR Imaging
}

BACKGROUND AND PURPOSE: Histopathologic studies have reported widespread cortical lesions in MS; however, in vivo detection by using routinely available pulse sequences is challenging. We investigated the relative frequency and subtypes of cortical lesions and their relationships to white matter lesions and cognitive and physical disability.

MATERIALS AND METHODS: Cortical lesions were identified and classified on the basis of concurrent review of 3D FLAIR and 3D T1-weighted IR-SPGR 3T MR images in 26 patients with MS. Twenty-five patients completed the MACFIMS battery. White matter lesion volume, cortical lesion number, and cortical lesion volume were assessed.

RESULTS: Overall, 249 cortical lesions were detected. Cortical lesions were present in 24/26 patients (92.3\%) (range per patient, 0-30; mean, $9.6 \pm 8.8$ ). Most $(94.4 \%, n=235)$ cortical lesions were classified as mixed cortical-subcortical (type I); the remaining $5.6 \%(n=14)$ were classified as purely intracortical (type II). Subpial cortical lesions (type III) were not detected. White matter lesion volume correlated with cortical lesion number and cortical lesion volume $\left(r_{\mathrm{S}}=0.652, r_{\mathrm{S}}=0.705\right.$, respectively; both $P<.001$ ). After controlling for age, depression, and premorbid intelligence, we found that all MR imaging variables (cortical lesion number, cortical lesion volume, white matter lesion volume) correlated with the SDMT score $\left(R^{2}=0.513, R^{2}=0.449, R^{2}=0.418\right.$, respectively; $\left.P<.014\right)$; cortical lesion number also correlated with the CVLT-II scores $\left(R^{2}=0.542-0.461, P<.043\right)$. The EDSS scores correlated with cortical lesion number and cortical lesion volume $\left(r_{\mathrm{S}}=0.472, r_{\mathrm{S}}=0.404\right.$, respectively; $P<.05)$, but not with white matter lesion volume.

CONCLUSIONS: Our routinely available imaging method detected many cortical lesions in patients with MS and was useful in their precise topographic characterization in the context of the gray matter-white matter junction. Routinely detectable cortical lesions were related to physical disability and cognitive impairment.

\begin{abstract}
ABBREVIATIONS: BVMT-R = Brief Visuospatial Memory Test-Revised; CES-D = Center for Epidemiologic Studies Depression Scale; CLN = cortical lesion number; CLV = cortical lesion volume; COWAT = Controlled Oral Word Association Test; CVLT-II = California Verbal Learning Test, second edition; D-KEFS = Delis-Kaplan Executive Function System Sorting Test; EDSS = Expanded Disability Status Scale; FLAIR = fluid-attenuated inversion recovery; IR-SPGR = inversion recovery spoiled gradient recalled; JLO = Judgment of Line Orientation Test; MACFIMS = Minimal Assessment of Cognitive Function in Multiple Sclerosis; Max = maximum; Min = minimum; MS = multiple sclerosis; $\mathrm{N} / \mathrm{A}=$ not applicable; NAART = North American Adult Reading Test; PASAT = Paced Auditory Serial Addition Test; $\mathrm{RR}=$ relapsing-remitting; $r_{\mathrm{S}}=$ Spearman correlation coefficient; $\mathrm{SDMT}=$ Symbol Digit Modalities Test; SP = secondary-progressive; St $\beta=$ partial regression coefficient for standardized data; $\mathrm{TLV}=$ total lesion volume $=\mathrm{CLV}+\mathrm{WMLV} ; \mathrm{WMLV}=$ white matter lesion volume
\end{abstract}

is a chronic progressive disease, characterized by a broad range of sensory-motor, cognitive, and neuropsy-

Received June 24, 2010; accepted after revision August 20

From the Center for Neurological Imaging (A.M., P.H., D.M., K.B., M.L., E.D., C.R.G.G.), Brigham and Women's Hospital, Harvard Medical School, Boston, Massachusetts; Partners Multiple Sclerosis Center (B.I.G., B.C.H., R.B.), Brigham and Women's Hospital, Harvard Medical School, Brookline, Massachusetts; Department of Radiology (P.H.), Lahey Clinic, Burlington, Massachusetts; Institute of Neurological Sciences (M.L.), National Research Council, Mangone, Cosenza, Italy; and the Biostatistics Center (B.C.H.), Department of Neurology, Massachusetts General Hospital, Boston, Massachusetts.

This work was supported by the National Multiple Sclerosis Society (grant RG3574A1). A. Mike, MD, receives support for her research activities from the Multiple Sclerosis International Federation with the McDonald Fellowship 2008.

Previously presented as a poster at: Annual Meeting of the American Academy of Neurology, Integrated Neuroscience Session of Multiple Sclerosis Imaging, April 10-17, 2010; Toronto, Ontario, Canada.

Please address correspondence to Charles R.G. Guttmann, MD, Center for Neurological Imaging, Brigham and Women's Hospital, Harvard Medical School, 221 Longwood Ave, RF 394, Boston, MA 02115; e-mail address: guttmann@bwh.harvard.edu

\section{indicates article with supplemental on-line table}

DOI 10.3174/ajnr.A2340 chiatric symptoms. Pathologically, MS affects the central nervous system with multifocal and diffuse inflammatory and neurodegenerative changes, but their exact etiology and pathogenesis remain uncertain. ${ }^{1}$ While early pathologic investigations describe involvement of both white matter and gray matter, ${ }^{2,3}$ white matter involvement has been studied more extensively. Recent advances in both histopathologic and imaging techniques have renewed the appreciation of gray matter involvement in MS. ${ }^{4}$ New histopathologic methods have found demyelinating lesions in significant portions of the cortex. ${ }^{5-10}$ In addition, neuroimaging techniques have detected structural and functional changes in the gray matter. ${ }^{4}$ Visualization of cortical lesions by MR imaging is challenging. ${ }^{8,10-12}$ Recently, novel MR imaging methods have been used to address these challenges, including novel pulse sequences, multichannel and high-resolution imaging, and ultra-high magnetic field strength. ${ }^{13-19}$ None of these techniques have demonstrated broad clinical applicability or vendor dissemination as applied to routine 3T MS imaging. In vivo assess- 


\begin{tabular}{|c|c|c|c|c|c|c|c|c|c|c|c|c|c|}
\hline \multicolumn{14}{|c|}{ Demographic, clinical, and MR imaging data of patients with $\mathbf{M S}^{\mathrm{a}}$} \\
\hline \multirow[b]{2}{*}{ Variables } & \multicolumn{3}{|c|}{ All Patients $(n=26)$} & \multicolumn{3}{|c|}{ Early RR $(n=7)$} & \multicolumn{3}{|c|}{ Late $\mathrm{RR}(n=13)$} & \multicolumn{3}{|c|}{$\mathrm{SP}(n=6)$} & \multirow{2}{*}{$\begin{array}{c}P \\
\text { Value }\end{array}$} \\
\hline & Min & Max & Mean & Min & Max & Mean & Min & Max & Mean & Min & Max & Mean & \\
\hline Age (yr) & 33 & 54 & $43.27 \pm 6.8$ & 33 & 48 & $39.43 \pm 6.2$ & 34 & 54 & $43.69 \pm 7.3$ & 42 & 54 & $46.83 \pm 4.4$ & \\
\hline $\begin{array}{l}\text { Disease duration } \\
\quad(\mathrm{yr})\end{array}$ & 2 & 28 & $13.27 \pm 7.8$ & 2 & 5 & $3.57 \pm 1.1$ & 10 & 28 & $16.38 \pm 5.8$ & 8 & 24 & $17.83 \pm 6.2$ & \\
\hline EDSS & 0.0 & 6.0 & $2.6 \pm 2.0$ & 0.0 & 3.5 & $1.4 \pm 1.2$ & 0.0 & 4.5 & $1.6 \pm 1.3$ & 3.5 & 6.0 & $5.2 \pm 1.3$ & \\
\hline Whole-brain CLN & 0 & 30 & $9.58 \pm 8.8$ & 0 & 23 & $6.00 \pm 7.9$ & 1 & 24 & $9.31 \pm 8.2$ & 0 & 30 & $14.33 \pm 10.3$ & .353 \\
\hline Type I No. & 0 & 26 & $9.04 \pm 8.3$ & 0 & 20 & $5.43 \pm 7.0$ & 0 & 24 & $9.00 \pm 8.2$ & 0 & 26 & $13.33 \pm 9.1$ & $\mathrm{~N} / \mathrm{A}$ \\
\hline Type II No. & 0 & 4 & $0.54 \pm 1.1$ & 0 & 3 & $0.57 \pm 1.1$ & 0 & 2 & $0.31 \pm 0.6$ & 0 & 4 & $1.00 \pm 1.7$ & $\mathrm{~N} / \mathrm{A}$ \\
\hline Right-sided CLN & 0 & 18 & $5.15 \pm 5.0$ & 0 & 12 & $3.00 \pm 4.2$ & 0 & 15 & $5.00 \pm 4.6$ & 0 & 18 & $8.00 \pm 6.2$ & $\mathrm{~N} / \mathrm{A}$ \\
\hline Left-sided CLN & 0 & 15 & $4.42 \pm 4.7$ & 0 & 11 & $3.00 \pm 4.0$ & 0 & 15 & $4.31 \pm 4.4$ & 0 & 15 & $6.33 \pm 5.9$ & $\mathrm{~N} / \mathrm{A}$ \\
\hline CLV (mL) & 0.000 & 0.692 & $0.161 \pm 0.21$ & 0.000 & 0.322 & $0.092 \pm 0.14$ & 0.004 & 0.652 & $0.157 \pm 0.20$ & 0.000 & 0.692 & $0.253 \pm 0.27$ & .382 \\
\hline WMLV (mL) & 0.54 & 51.47 & $15.22 \pm 13.8$ & 1.12 & 32.49 & $7.69 \pm 11.2$ & 0.54 & 51.47 & $16.89 \pm 14.00$ & 3.49 & 41.98 & $20.38 \pm 14.6$ & .120 \\
\hline TLV (mL) & 0.55 & 51.93 & $15.38 \pm 13.94$ & 1.14 & 32.81 & $7.78 \pm 11.31$ & 0.55 & 51.93 & $17.04 \pm 14.10$ & 3.49 & 42.67 & $20.64 \pm 14.86$ & .132 \\
\hline
\end{tabular}

${ }^{a}$ Male/female ratio (percentage) for all patients is 19/7 (73/27); Early RR, 4/3 (57/43); Late RR, 10/3 (77/23), and SP, 5/1 (83/17).

${ }^{b}$ Comparison of the 3 patient subgroups (Kruskal-Wallis test).

ment of cortical lesions is of great significance because the pathologic processes that have taken place in the gray matter and their relationship to white matter pathology and gray matter atrophy and clinical outcomes will improve our understanding of MS pathogenesis. ${ }^{20,21}$

In the present study, we used a high-resolution $3 \mathrm{~T}$ brain MR imaging protocol that combined a multiplanar display of 3D FLAIR and T1-weighted 3D IR-SPGR sequences. This approach took advantage of the high contrast sensitivity of FLAIR for imaging cortical lesions combined with IR-SPGR to delineate the boundary between the cortex and white matter. These sequences are widely available on clinical scanners from multiple vendors and can be integrated in clinical routine with reasonable scanning time. We aimed to evaluate the ability of this standard protocol to depict cortical lesions and their different subtypes and to explore the relationships between cortical lesions versus white matter lesion load, cognitive dysfunction, and physical disability in MS.

\section{Materials and Methods}

\section{Patient Population}

Twenty-six patients with MS according to the McDonald criteria ${ }^{22}$ were recruited from the Partners Multiple Sclerosis Center at Brigham and Women's Hospital, Boston, Massachusetts. Each patient had a clinical examination, neuropsychological test, and brain MR imaging. The interval between clinical examination and neuropsychological testing ranged between 0 and 126 days (mean, $28.2 \pm 28.8$ days) and between MR imaging and neuropsychological testing ranged between 0 and 21 days $(1.8 \pm 5.0$ days $)$. We studied patients with 3 different clinical subtypes: 1$)$ Early RR ( $n=$ $7)$, RR disease duration $\leq 5$ years; 2$)$ Late $\mathrm{RR}(n=13)$, RR disease duration $\geq 10$ years; 3$)$ SP $(n=6)$, SP disease course with at least 3 years' duration in this stage. Clinical and demographic characteristics are presented in Table 1. Patients with a history of alcohol or drug dependence, major psychiatric illness, neurologic disease (other than MS), or gross visual impairment were excluded. Physical disability was measured with the EDSS. ${ }^{23}$ None of the patients were cognitively tested or scanned until at least 1 month had elapsed following an exacerbation. At the time of the study, 22 patients $(84.6 \%)$ had received immunomodulatory or immunosupressive therapy (glatiramer acetate, interferon $\beta-1 \mathrm{~b}$, interferon $\beta-1 \mathrm{a}$, mitoxantrone, methotrexate, mycophenolate mofetil, dacli- zumab, or intravenous immunoglobulin). The study was approved by the institutional review board, and all patients provided written informed consent.

\section{Neuropsychological Testing}

Twenty-five patients with MS completed the MACFIMS, a test battery that has been shown to be sensitive to cognitive functions commonly impaired in MS. ${ }^{24}$ MACFIMS is composed of the following tests: 1 ) The PASAT measures working memory and speed of information processing. Subjects hear a series of digits every 2 seconds and are required to add sequential pairs so that each digit is added to the digit immediately preceding it. The score is the total number of correct responses. 2) The SDMT is a measure of working memory and speed of information processing that requires subjects to substitute numbers for symbols according to a look-up table. The score is the total number of correct items. 3) The CVLT-II measures verbal learning and memory by using a 16-item word list. There are 5 learning trials. The CVLT-II uses 2 scores: CVLT-II T1-T5, which is total recall across the 5 learning trials and CVLT-II Long-Delay Free Recall, which is recall after a 25-minute delay. 4) The BVMT-R tests spatial learning and memory by requiring subjects to learn a matrix of 6 simple abstract designs. It calculates 2 scores: Total Recall, which is the total score across the 3 learning trials, and Delayed Recall, which is recall after a 25-minute delay. 5) The D-KEFS assesses executive function by requiring subjects to sort cards by using perceptual stimuli and printed words. The score is the number of Confirmed Correct Sorts. 6) The JLO estimates visual perception and spatial processing. Subjects are required to match the angle defined by 2 stimulus lines. The score is the total number of correct responses. 7) The COWAT tests verbal fluency by asking subjects to generate as many words as they can that begin with 3 stimulus letters. The score is the total number of words generated.

The NAART was used to estimate premorbid intelligence. ${ }^{25}$ Depressive symptoms were assessed by the CES-D. ${ }^{26}$ Cognitive tests were conducted in a single session by a trained research assistant (K.B.) under the supervision of an experienced clinical neuropsychologist (B.I.G.). Both were blinded to MR imaging findings.

\section{MR Imaging}

Acquisition. High-resolution brain MR images were acquired by using 2 pulse sequences on a 3T Signa scanner (GE Healthcare, Milwaukee, Wisconsin) with an 8-channel head coil: T1-weighted 3D IR-SPGR (138 contiguous 1 -mm-thick sagittal sections, TR/TE = 
$7.48 / 2.984 \mathrm{~ms}, \mathrm{TI}=450 \mathrm{~ms}$, matrix size $=256 \times 256$, in-plane pixel spacing $=1 \times 1 \mathrm{~mm}, \mathrm{FOV}=256 \times 256 \mathrm{~mm}$, flip angle $=15^{\circ}$, scanning time $\sim 9$ minutes) and single-slab $3 \mathrm{D}$ fast spin-echo FLAIR (136 contiguous $1.2-\mathrm{mm}$-thick sagittal sections, TR/TE $=6200 / 200$ $\mathrm{ms}, \mathrm{TI}=1901 \mathrm{~ms}$, matrix size $=256 \times 256$, in-plane pixel spacing $=$ $1.2 \times 1.2 \mathrm{~mm}, \mathrm{FOV}=307 \times 307 \mathrm{~mm}$, flip angle $=90^{\circ}$, scanning time $\sim 6$ minutes).

\section{MR Imaging Analysis}

Cortical Lesion Detection and Typing. Image interpretation was performed by a neurologist with clinical and imaging experience in MS (A.M.) in collaboration with a Certificate of Added Qualification-certified neuroradiologist (P.H.). Both raters were blinded to clinical and neuropsychiatric findings. The 3D IR-SPGR and FLAIR images were spatially coregistered for concordant image analysis by using the Functional Magnetic Resonance Imaging of the Brain's Linear Image Registration Tool (http://www.fmrib.ox.ac.uk/ flirt). ${ }^{27}$ This step required approximately 5 minutes of computer time. OsiriX software (Version 3.3, http://www.osirix-viewer.com/ Downloads.html) was used to view the coregistered FLAIR and IR-SPGR images concurrently in multiple reformatted planes. Initially, hyperintense signal-intensity abnormalities in the proximity of the cortical mantle were detected on the FLAIR sequence. To be counted, a lesion had to have a minimum diameter of $3 \mathrm{~mm}$ in any of 3 orthogonal views. Each lesion detected on FLAIR was then also required to demonstrate hypointensity on the IR-SPGR sequence. In addition, the lesion was required to involve the cerebral cortex with or without involvement of underlying white matter as defined on IRSPGR images. Lesions involving only the subcortical white matter at the gray matter-white matter junction without extension into the cortical mantle (juxtacortical lesions) were excluded. Precise visualization of the spatial extension of cortical lesions was enabled by the 3D IR-SPGR isotropic images (Figs 1 and 2).

Cortical lesion classification was based on a histopathologic scheme $^{8}$ : A type I lesion involved both the cortex and adjoining subcortical white matter (mixed gray matter-white matter lesion), a type II lesion was restricted purely to the cortex (intracortical lesion), and a type III lesion was bandlike, extending from the pial surface into the cortex (subpial lesion).

During quality control, we found 7 cases (27\%) with some degree of degradation related to motion or flow artifacts. However, the quality of all images was sufficient for our analysis. We registered each patient's total cortical lesion number and the number of lesions assigned to each subtype. We also distinguished cortical lesions in the left-versus-right cerebral hemispheres. Cortical lesion detection and characterization required 4-6 hours/examination of expert analyst time, depending on the lesion load.

The 2 raters reviewed all lesions they identified and came to a consensus with regard to number and lesion type. Five cases were randomly chosen from the major clinical subgroups (1 Early RR, 2 Late RRs, 2 SPs) to test intra- (A.M.) and inter-rater (A.M., P.H.) variability of cortical lesion number assessment. For both evaluations, the coefficient of variation was defined as the SD divided by the mean. The mean inter-rater variability was $4.47 \%$ for total cortical lesion number and $4.85 \%$ for type I cortical lesion number. The mean intrarater variability was $4.53 \%$ for total cortical lesion number and $4.21 \%$ for type I cortical lesion number. Reproducibility of type II lesion evaluation was not analyzed separately due to its small proportion (see "Results").

\section{Lesion-Load Measurement}

Field inhomogeneity correction was performed by applying an automated algorithm based on entropy minimization, ${ }^{28}$ requiring approximately 5 minutes of computer time. To estimate white matter lesion volume and cortical lesion volume from FLAIR images, we outlined lesions by using a semiautomated thresholding technique with the software 3DSlicer (Version 3.4, http://www.slicer.org). The method relies on user-guided specification of the intensity threshold range, which is manually adjustable during lesion segmentation. The algorithm labels all pixels with signal intensity within the set threshold range in the lesion area identified manually by the user. When outlining lesions in the cortex, we took care not to include FLAIR hyperintense signal-intensity abnormalities, which did not have corresponding IR-SPGR hypointensities. Lesion volume determination by an expert required $20-40$ minutes/case.

\section{Statistical Analysis}

All statistical analyses were performed by using the Statistical Package for the Social Sciences software, Version 13.0 (SPSS, Chicago, Illinois). Prevalence of lesions in each hemisphere was compared using the Wilcoxon signed rank test. Nonparametric tests were used because distributions of the MR imaging variables were not normal. The Kruskal-Wallis test was used for comparisons among the 3 patient subgroups. If a significant difference was observed, the Mann-Whitney $U$ test was performed for pair-wise comparisons, and a Bonferroni correction was used to account for multiple comparisons. The nonparametric Spearman rank correlation test was applied to investigate univariate correlations among the $3 \mathrm{MR}$ imaging measures (cortical lesion number, cortical lesion volume, white matter lesion volume), neuropsychological test performance, and physical disability. In addition, multiple linear regression was used to assess the cognitive-MR imaging relationship, adjusting for age, premorbid intelligence quotient (NAART), and depressive symptoms (CES-D). Due to small sample sizes, the univariate modeling was not performed within individual clinical subgroups. A 2-tailed level of $P<.05$ was the significance threshold.

\section{Results}

\section{Cortical Lesion Frequency and Typing}

Descriptive statistics of the MR imaging data are listed in the Table. Overall, 249 cortical lesions were detected in $24 / 26$ patients $(92.3 \%)$. Lesions per patient ranged between 0 and 30; mean, $9.6 \pm 8.8$. Two hundred thirty-five lesions $(94.4 \%)$ were classified as type I; 14 (5.6\%), as type II; and none, as type III. All 24 patients with cortical lesions had type I lesions, while the 14 type II cortical lesions were confined to 7 patients (2 Early RRs, 3 Late RRs, 2 SPs). No statistically significant hemispheric differences in cortical lesion number were found.

\section{Comparison of the Clinical Subgroups}

Mean and median values of cortical lesion number, cortical lesion volume, and white matter lesion volume were higher inLate RR compared with Early RR and higher in SP compared with the Late RR subgroup; however, these differences were not statistically significant (Table).

A statistically significant difference in the BVMT-R Total 
Sagittal

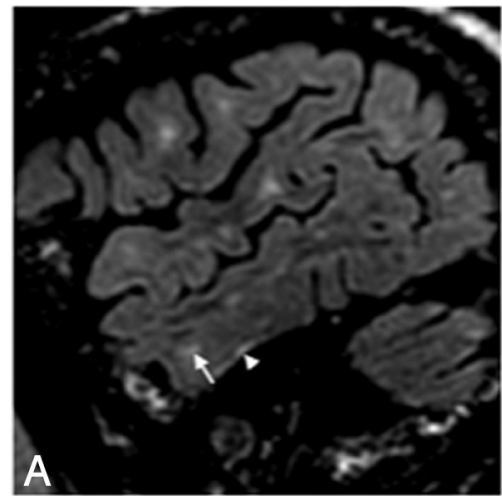

FLAIR

SPGR
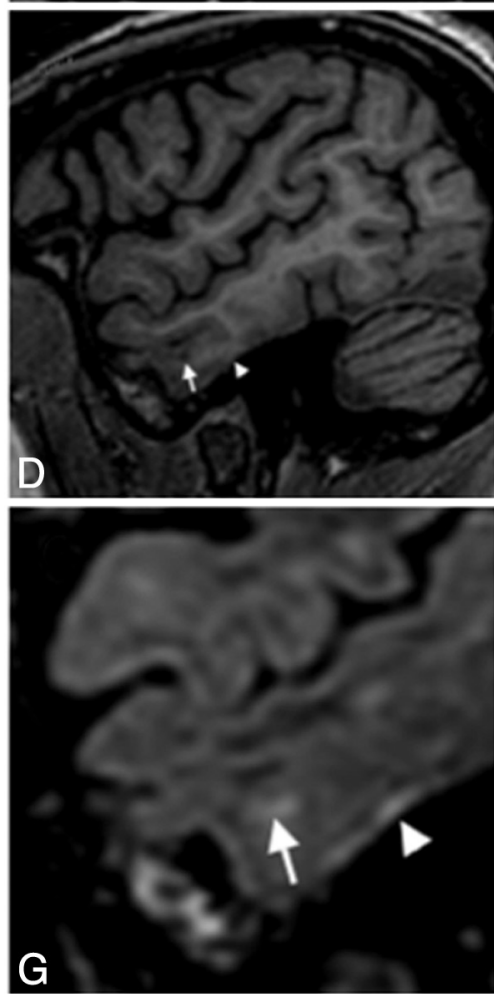

FLAIR

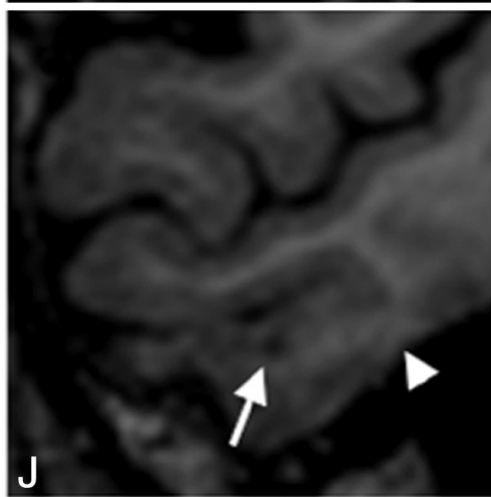

Axial
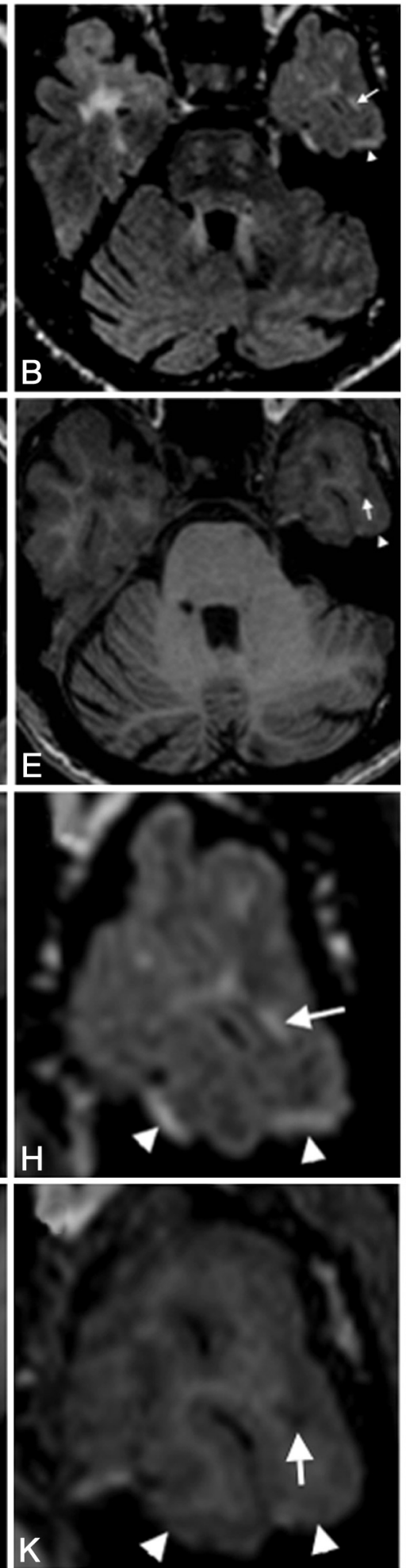

\section{Coronal}
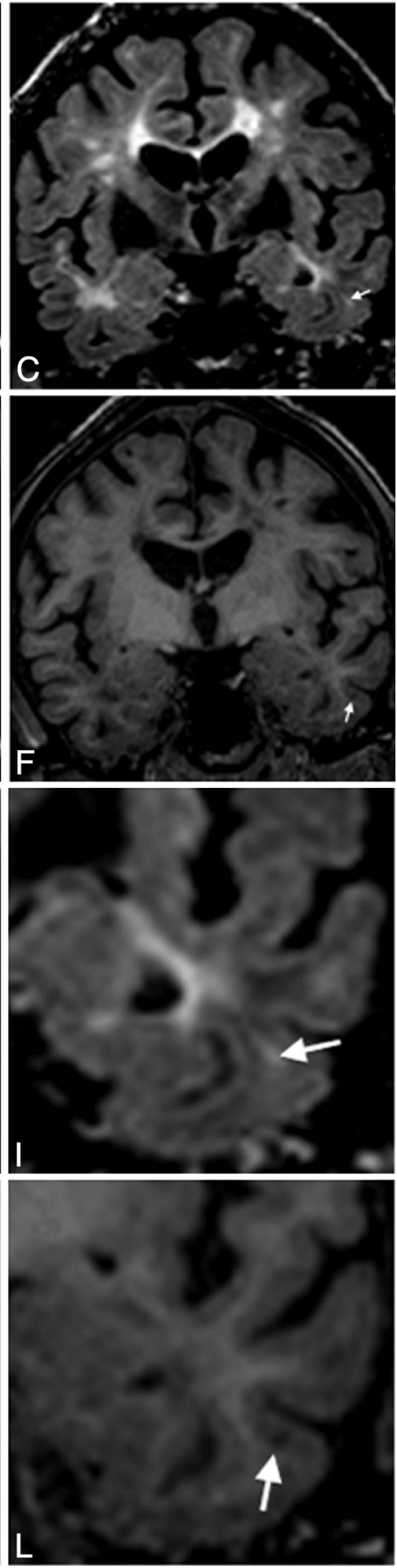

Fig 1. Type I cortical lesion shown on multiplanar images. $A-F$, Unmagnified images. $G-H$, Magnified images. The same type I lesion (arrows) is shown on FLAIR ( $A-C$, $G-I)$ and IR-SPGR ( $D-F$ $J-L)$ images, reconstructed in sagittal $(A, D, G, J)$, axial $(B, E, H, K)$, and coronal $(C, F, I, L)$ planes. Note that the lesion is conspicuous on FLAIR, while its anatomic localization is well-defined on the IR-SPGR images. On the IR-SPGR images, gray matter involvement is clear on the sagittal and coronal views, while white matter involvement is visible on the sagittal and axial views, Arrowheads indicate FLAIR hyperintense areas without a corresponding hypointensity on the IR-SPGR images, suggesting the possibility of artifacts; these were not counted as cortical lesions.

Recall was observed among the 3 subgroups (Kruskal-Wallis, $P=.04$ ). For pair-wise comparisons, patients with SP per- formed worse than those with Late RR on BVMT-R Total Recall (Bonferroni-corrected, $P=.029$ ). 

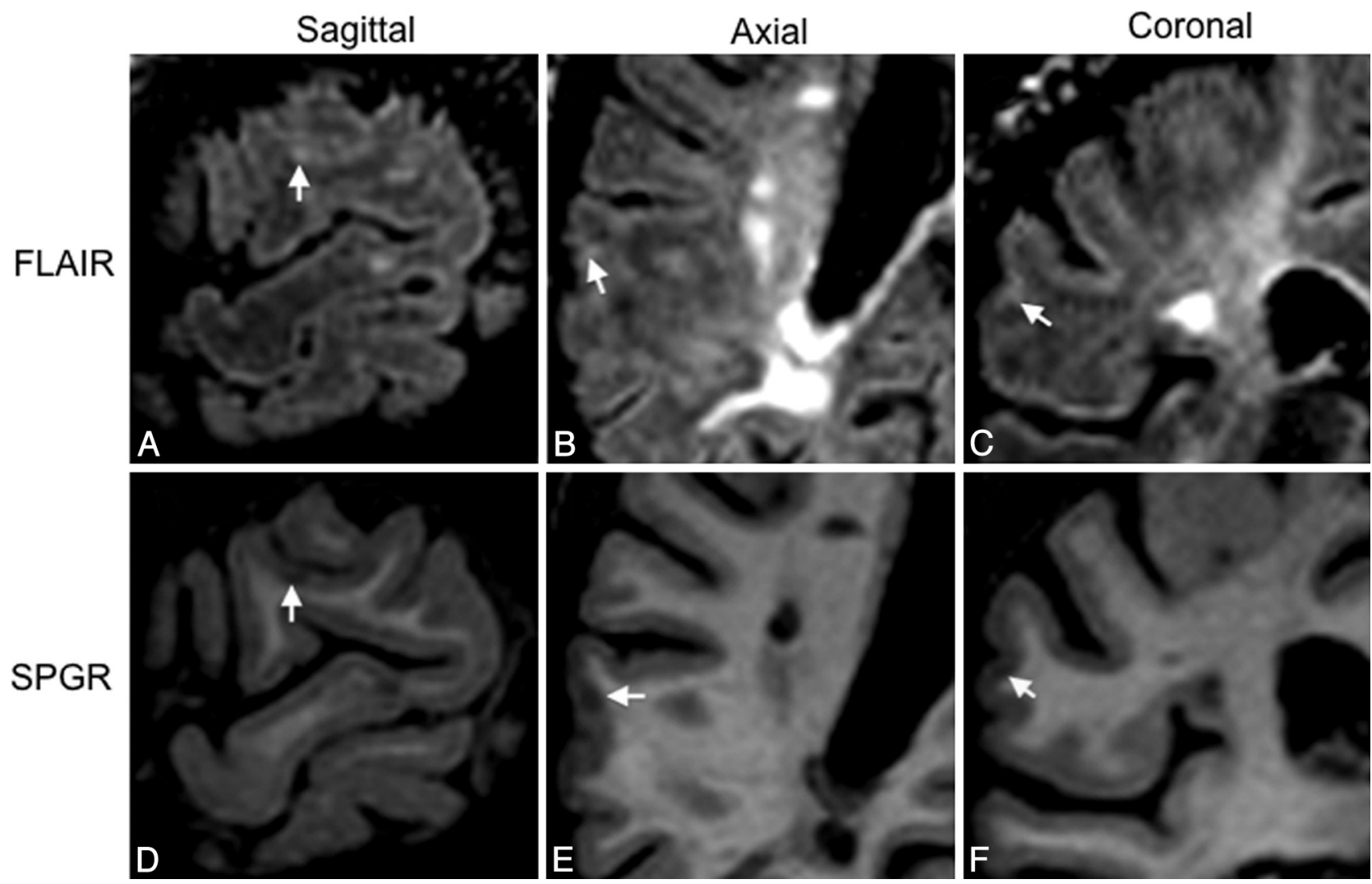

Fig 2. Type II cortical lesion shown on multiplanar images. A type II lesion (arrows) is shown on FLAIR (upper row) and IR-SPGR (bottom row). $A$ and $D$, Sagittal view. B and $E$, Axial view. $C$ and $F$, Coronal view. Note that the lesion is confined to the cerebral cortical gray matter and does not involve the subcortical white matter, which is clearly seen in the 3 orthogonal planes of the IR-SPGR images.

\section{Associations among Cortical Lesions, White Matter Lesions, Physical Disability, and Cognitive Performances} Results of univariate correlation analyses between cortical lesions and white matter lesion volume as well as between these lesional metrics versus physical disability and neuropsychological test performance are summarized in the On-line Table. Cortical lesion volume and cortical lesion number showed significant correlations with white matter lesion volume. EDSS score significantly correlated with cortical lesion number and cortical lesion volume, but not with white matter lesion volume. In univariate analyses, all $3 \mathrm{MR}$ imaging variables (cortical lesion number, cortical lesion volume, white matter lesion volume) correlated with SDMT. Cortical lesion number correlated with both immediate and delayed CVLT-II scores. Cortical lesion volume and white matter lesion volume correlated with both immediate and delayed BVMT-R scores. None of the MR imaging variables correlated with PASAT, D-KEFS, JLO, or COWAT. After we corrected for age, depression, and premorbid intelligence quotient in multiple linear regression analyses, SDMT remained associated with cortical lesion number (overall $R^{2}=0.513$, St $\beta=-0.566, P=.002$ ), cortical lesion volume (overall $R^{2}=0.449$, St $\beta=-0.492, P=.008$ ), and white matter lesion volume (overall $R^{2}=0.418$, St $\beta=$ $-0.461, P=.014)$. Cortical lesion number remained associated with CVLT-II T1-T5 (overall $R^{2}=0.542$, St $\beta=-0.421$, $P=.013$ ) and CVLT-II Long-Delayed Free Recall (overall $R^{2}$ $=0.461$, St $\beta=-0.363, P=.043)$. However in the final adjusted models, MR imaging variables no longer correlated with BVMT-R.

\section{Discussion}

Using a clinically applicable MR imaging method combining 3D FLAIR and IR-SPGR sequences, we assessed cortical lesions in patients with MS. The detected cortical lesion load is comparable with the bulk of cortical lesions assessed by specialized MR imaging methods developed for sensitive cortical lesion delineation. ${ }^{13,15,16,18,29}$ While it has been reported that cortical lesions are more conspicuous on 3D double inversion recovery compared with 3D-FLAIR images, ${ }^{13,17}$ the former is not yet widely available on clinical scanners. FLAIR hyperintensities might represent lesions, perivenular spaces, or CSFrelated flow artifacts. Our lesion detection required that any FLAIR hyperintense lesions also show concurrent hypointensity on IR-SPGR images. This rule should have minimized the inclusion of nonlesional hyperintensities (eg, artifacts, Fig 1). The possibility of misclassifying small microischemic lesions as MS lesions exists with our applied method; however, the prevalence of ischemic lesions was likely low, given the relatively young age of our MS cohort. Our dual image-based cortical lesion identification method leads to a trade-off with higher specificity and lower sensitivity than conventional methods.

We detected cortical lesions in $92.3 \%$ of patients, supporting the notion that focal cortical lesions are highly prevalent in MS. ${ }^{6,11,13-16,30}$ Cortical lesions occurred in all studied clinical subgroups, including $85.7 \%$ of patients with Early RR MS. These results are consistent with recent MR imaging studies that detected cortical lesions from the earliest clinical stages of MS, even in patients with clinically isolated syn- 
drome. ${ }^{13,14,30-32}$ We found a trend toward higher number and volume of cortical lesions with disease duration and progression when comparing Early RR versus Late RR versus SP subgroups (Table). However, these differences did not reach statistical significance, possibly because of small sample sizes. Technical factors might also influence lesion detection: Images from more disabled patients may contain more motion artifacts, and in more advanced disease, diffuse cortical damage may blur the contrast between gray matter and white matter, ${ }^{33}$ thereby obscuring juxtacortical and cortical-subcortical lesions. Both of these factors could result in underestimation of cortical lesions in SPMS. Several histopathologic and MR imaging studies have found an increasing number of cortical lesions with advancing disease duration and clinical stage. ${ }^{7,30-32}$ One study was unable to confirm these findings, ${ }^{14}$ and a 7T MR imaging study showed differences between patients with RRMS and SPMS only for subpial lesions. ${ }^{18}$

We classified most cortical lesions as the corticosubcortical type (type I), while intracortical lesions (type II) made up $5.63 \%$, and no subpial lesions (type III) were detected. In histopathologic studies, subpial lesions were the most prevalent type (prevalence, 44\%-70\%), followed by corticosubcortical lesions (10\%-34\%) and small intracortical lesions $(13 \%-26 \%))^{5-9}$ We believe that we had much higher sensitivity in detecting type I lesions versus both type II and type III lesions. This resulted in our disproportionate estimation of the distribution of lesion subtypes, by using histologic reports as the standard of reference. There are probably 2 major reasons for this: Type II (intracortical) lesions are relatively small and difficult to detect even at the $\sim 1-\mathrm{mm}$ isotropic image resolution used in our protocol. Type III lesions escaped our detection most likely because severe subpial cortical tissue damage results in MR signal intensity closely approximating that of the adjacent subarachnoid CSF, limiting their conspicuity on IR-SPGR. Histopathologically, the intensity of inflammation in corticosubcortical lesions is higher than that in lesions confined purely to the cortex, resulting in a more conspicuous MR imaging appearance. ${ }^{8}$

A further complicating factor relates to the different cytoarchitecture of the cortex compared with white matter, in that subpial lesions are located in the myelin-sparse upper layer of the cortex. ${ }^{6,8,10,12}$ While ultra-high-field MR imaging allowed depiction of subpial lesions, ${ }^{18,19}$ previous MR imaging studies at field strengths $<4 \mathrm{~T}$ were similarly to our method unable to visualize them. ${ }^{14-17,34}$ The multiplanar display of our isotropic resolution IR-SPGR images allowed precise anatomic lesion classification in relation to the gray matter-white matter junction and cortical folding patterns. We believe this is a critical step in assigning lesions to their proper subtype. Thus, our method might have more accurately classified lesions as type I, while previous methods would have classified these lesions as type II or juxtacortical (Figs 1 and 2).

Of fundamental importance is whether similar pathologic processes drive focal lesion formation in the white and gray matter. ${ }^{6-9,20,21}$ In our study, cortical lesion measures reflected nearly entirely cortical-subcortical types and showed moderate correlations with white matter lesion volume, suggesting that the underlying pathogenic processes might be related. The relationship between inflammatory demyelinating lesions in white matter and gray matter has previously demonstrated con- flicting results: Some studies found correlation, ${ }^{30,32,35,36}$ whereas others did not. ${ }^{18,31,37}$ Unfortunately, the approach we used did not shed light on the relationship with subpial lesions.

In univariate analysis, cortical lesion metrics showed significant correlations with EDSS scores, while white matter lesion volume did not, though a trend was apparent. A large cross-sectional study by using a $1.5 \mathrm{~T} 2 \mathrm{D}$ double inversion recovery sequence showed correlation between cortical lesion number and EDSS scores in patients with RRMS, SPMS, and clinically isolated syndrome. ${ }^{30}$ Applying the same MR imaging method, a 2-year longitudinal study reported the predictive value of baseline cortical lesion load on the progression of physical disability in primary progressive MS patients, ${ }^{35}$ and during a 3-year follow-up in patients with RRMS and SPMS. ${ }^{37}$ However, no correlation was found between physical disability and cortical lesion load by using other imaging methods. ${ }^{14,15}$ Subpial lesions correlated with physical disability, but total cortical lesions did not, using 7T MR imaging. ${ }^{18}$ Larger studies enabling multivariate regression analysis of cortical lesion subtypes and white matter lesions will be necessary to understand the specific contribution of cortical lesion subtypes toward clinical outcomes.

We explored the relationship between cognitive performance and lesion load in white matter and cortical gray matter compartments of patients with MS. Very few studies have examined this previously. ${ }^{32,36}$ Therefore, we wanted to assess a broad range of neuropsychological variables, which could potentially include cortical contributions (eg, information-processing speed, new learning, verbal and nonverbal memory, and executive function). After we controlled for age, depression, and premorbid intelligence, cortical lesion number, cortical lesion volume, and white matter lesion volume independently predicted the performance of information-processing speed and working memory (SDMT). In addition, cortical lesion number also predicted verbal learning and memory (CVLT-II). One limitation of our study is the lack of cognitive data on healthy controls. Thus, we cannot ascertain the prevalence and severity of cognitive impairment in our cohort.

Previous studies demonstrated that information-processing speed, working memory, and verbal memory are commonly affected in MS. ${ }^{24,38,39}$ Performance on SDMT involves diverse mental functions, including complex scanning and visual tracking, requiring integrity of widely dispersed cortical regions. ${ }^{39}$ The PASAT also measures information-processing speed and working memory; however, compared with SDMT, lower accuracy and sensitivity have been found in predicting cognitive deficits in $\mathrm{MS},{ }^{39}$ and weaker correlations with MR imaging metrics have been previously reported. ${ }^{40-42}$

Our findings suggesting the importance of cortical lesions with regard to CVLT-II are in line with previous studies showing associations between gray matter atrophy and verbal learning. ${ }^{43,44}$

The absence of a significant correlation between cortical lesions and other cognitive tests (BVMT-R, COWAT, JLO, and D-KEFS) may be related to their lower sensitivity in showing associations with distributed multiple lesions ${ }^{39-41,45}$; however, regional cortical lesions were not assessed in our study. Other factors may have contributed to our failure to find significant correlations across the spectrum of cognitive domains commonly affected in MS: The small sample size and the high 
proportion of patients with RRMS compared with SPMS may haye limited the severity of the deficits observed.

OUR FINDINGS SUGGEST THAT BOTH WHITE AND GRAY MATTER LESIONS INFLUENCE COGNITIVE PERFORMANCE IN MS. HOWEVER, CORTICAL LESIONS MAY HAVE A PARTICULARLY IMPORTANT CONTRIBUTION TO THIS RELATIONSHIP, BECAUSE BESIDES INFORMATION-PROCESSING-SPEED PERFORMANCE, CORTICAL LESION NUMBER WAS ALSO SIGNIFICANTLY CORRELATED WITH VERBAL LEARNING ABILITIES. TWO RECENT STUDIES USING A DOUBLE INVERSION RECOVERY SEQUENCE YIELDED SIMILAR RESULTS. ${ }^{32,36}$ Future studies using this clinically applicable MR imaging protocol in a larger sample size and in a longitudinal fashion may further extend our knowledge about the pathogenic and clinical relevance of cortical lesions in MS, even though there remains a prominent need for clinically applicable MR imaging methodology capable of clearly depicting subpial lesions in MS

\section{Conclusions}

The combination of 3T high-resolution T2-FLAIR and T1-IRSPGR MR imaging detected many cortical lesions and allowed precise anatomic lesion classification. Our data suggest that focal cortical demyelination is a significant contributor to both the physical disability and cognitive decline in patients with MS.

\section{Acknowledgments}

We are grateful for the support of the clinical staff at the Partners Multiple Sclerosis Center.

\section{References}

1. Trapp BD, Peterson J, Ransohoff RM, et al. Axonal transection in the lesions of multiple sclerosis. $N$ Engl J Med 1998;338:278-85

2. Lumsden C. The Neuropathology of Multiple Sclerosis. Amsterdam: Elsevier; 1970

3. Brownell B, Hughes JT. The distribution of plaques in the cerebrum in multiple sclerosis. J Neurol Neurosurg Psychiatry 1962;25:315-20

4. Pirko I, Lucchinetti CF, Sriram S, et al. Gray matter involvement in multiple sclerosis. Neurology 2007;68:634-42

5. Albert M, Antel J, Bruck W, et al. Extensive cortical remyelination in patients with chronic multiple sclerosis. Brain Pathol 2007;17:129-38

6. Bo L, Vedeler CA, Nyland HI, et al. Subpial demyelination in the cerebral cortex of multiple sclerosis patients. J Neuropathol Exp Neurol 2003;62:723-32

7. Kutzelnigg A, Lucchinetti CF, Stadelmann C, et al. Cortical demyelination and diffuse white matter injury in multiple sclerosis. Brain 2005;128:2705-12

8. Peterson JW, Bo L, Mork S, et al. Transected neurites, apoptotic neurons, and reduced inflammation in cortical multiple sclerosis lesions. Ann Neurol 2001;50:389-400

9. Wegner C, Esiri MM, Chance SA, et al. Neocortical neuronal, synaptic, and glial loss in multiple sclerosis. Neurology 2006;67:960-67

10. Kidd D, Barkhof F, McConnell R, et al. Cortical lesions in multiple sclerosis. Brain 1999;122(pt 1):17-26

11. Geurts JJ, Bo L, Pouwels PJ, et al. Cortical lesions in multiple sclerosis: combined postmortem MR imaging and histopathology. AJNR Am J Neuroradiol 2005;26:572-77

12. Bakshi R, Ariyaratana S, Benedict RH, et al. Fluid-attenuated inversion recovery magnetic resonance imaging detects cortical and juxtacortical multiple sclerosis lesions. Arch Neurol 2001;58:742-48

13. Simon B, Schmidt S, Lukas $\mathrm{C}$, et al. Improved in vivo detection of cortical lesions in multiple sclerosis using double inversion recovery MR imaging at 3 Tesla. Eur Radiol 2010;20:1675-83. Epub 2010 Jan 22

14. Bagnato F, Butman JA, Gupta S, et al. In vivo detection of cortical plaques by MR imaging in patients with multiple sclerosis. AJNR Am J Neuroradiol 2006;27:2161-67

15. Nelson F, Poonawalla AH, Hou P, et al. Improved identification of intracortical lesions in multiple sclerosis with phase-sensitive inversion recovery in combination with fast double inversion recovery MR imaging. AJNR Am J Neuroradiol 2007;28:1645-49

16. Nelson F, Poonawalla A, Hou P, et al. 3D MPRAGE improves classification of cortical lesions in multiple sclerosis. Mult Scler 2008;14:1214-19

17. Geurts JJ, Pouwels PJ, Uitdehaag BM, et al. Intracortical lesions in multiple sclerosis: improved detection with 3D double inversion-recovery MR imaging. Radiology 2005;236:254-60

18. Mainero $\mathrm{C}$, Benner $\mathrm{T}$, Radding $\mathrm{A}$, et al. In vivo imaging of cortical pathology in multiple sclerosis using ultra-high field MRI. Neurology 2009;73:941-48

19. Kangarlu A, Bourekas EC, Ray-Chaudhury A, et al. Cerebral cortical lesions in multiple sclerosis detected by MR imaging at 8 Tesla. AJNR Am J Neuroradiol 2007;28:262-66

20. Chard D, Miller D. Grey matter pathology in clinically early multiple sclerosis: evidence from magnetic resonance imaging. J Neurol Sci 2009;282:5-11

21. Geurts JJ, Stys PK, Minagar, et al. Gray matter pathology in (chronic) MS modern views on an early observation. J Neurol Sci 2009;282:12-20

22. Polman $\mathrm{CH}$, Reingold SC, Edan G, et al. Diagnostic criteria for multiple sclerosis: 2005 revisions to the "McDonald Criteria." Ann Neurol 2005;58:840-46

23. Kurtzke JF. Rating neurologic impairment in multiple sclerosis: an expanded disability status scale (EDSS). Neurology 1983;33:1444-52

24. Benedict RH, Cookfair D, Gavett R, et al. Validity of the minimal assessment of cognitive function in multiple sclerosis (MACFIMS). J Int Neuropsychol Soc 2006;12:549-58

25. Uttl B. North American Adult Reading Test: age norms, reliability, and validity. J Clin Exp Neuropsychol 2002;24:1123-37

26. Radloff L. CES-D scale: a self report depression scale for research in the general population. Applied Psychological Measurement 1977;1:385-401

27. Jenkinson M, Smith S. A global optimisation method for robust affine registration of brain images. Med Image Anal 2001;5:143-56

28. Sled JG, Zijdenbos AP, Evans AC. A nonparametric method for automatic correction of intensity nonuniformity in MRI data. IEEE Trans Med Imaging 1998;17:87-97

29. Calabrese M, Filippi M, Rovaris M, et al. Morphology and evolution cortical lesions in multiple sclerosis: a longitudinal MRI study. Neuroimage 2008;42:1324-28

30. Calabrese M, De Stefano N, Atzori M, et al. Detection of cortical inflammatory lesions by double inversion recovery magnetic resonance imaging in patients with multiple sclerosis. Arch Neurol 2007;64:1416-22

31. Calabrese M, Filippi M, Rovaris M, et al. Evidence for relative cortical sparing in benign multiple sclerosis: a longitudinal magnetic resonance imaging study. Mult Scler 2009;15:36-41

32. Calabrese M, Agosta F, Rinaldi F, et al. Cortical lesions and atrophy associated with cognitive impairment in relapsing-remitting multiple sclerosis. Arch Neurol 2009;66:1144-50

33. Chen JT, Narayanan S, Collins DL, et al. Relating neocortical pathology to disability progression in multiple sclerosis using MRI. Neuroimage 2004;23:1168-75

34. Geurts JJ, Blezer EL, Vrenken H, et al. Does high-field MR imaging improve cortical lesion detection in multiple sclerosis? J Neurol 2008;255:183-91

35. Calabrese M, Rocca MA, Atzori M, et al. Cortical lesions in primary progressive multiple sclerosis: a 2-year longitudinal MR study. Neurology 2009;72: $1330-36$

36. Roosendaal SD, Moraal B, Pouwels PJ, et al. Accumulation of cortical lesions in MS: relation with cognitive impairment. Mult Scler 2009;15:708-14

37. Calabrese M, Rocca MA, Atzori M, et al. A 3-year magnetic resonance imaging study of cortical lesions in relapse-onset multiple sclerosis. Ann Neurol 2010;67:376-83

38. Chiaravalloti ND, DeLuca J. Cognitive impairment in multiple sclerosis. Lancet Neurol 2008;7:1139-51

39. Strober L, Englert J, Munschauer F, et al. Sensitivity of conventional memory tests in multiple sclerosis: comparing the Rao Brief Repeatable Neuropsychological Battery and the Minimal Assessment of Cognitive Function in MS. Mult Scler 2009;15:1077-84

40. Christodoulou C, Krupp LB, Liang Z, et al. Cognitive performance and MR markers of cerebral injury in cognitively impaired MS patients. Neurology 2003;60:1793-98

41. Benedict RH, Weinstock-Guttman B, Fishman I, et al. Prediction of neuropsychological impairment in multiple sclerosis: comparison of conventional magnetic resonance imaging measures of atrophy and lesion burden. Arch Neurol 2004;61:226-30

42. Lazeron RH, Boringa JB, Schouten $\mathrm{M}$, et al. Brain atrophy and lesion load as explaining parameters for cognitive impairment in multiple sclerosis. Mult Scler 2005;11:524-31

43. Sanfilipo MP, Benedict RH, Weinstock-Guttman B, et al. Gray and white matter brain atrophy and neuropsychological impairment in multiple sclerosis. Neurology 2006;66:685-92

44. Amato MP, Bartolozzi ML, Zipoli V, et al. Neocortical volume decrease in relapsing-remitting MS patients with mild cognitive impairment. Neurology 2004;63:89-93

45. Sanchez MP, Nieto A, Barroso J, et al. Brain atrophy as a marker of cognitive impairment in mildly disabling relapsing-remitting multiple sclerosis. Eur J Neurol 2008;15:1091-99 